\title{
Lipoprotéine lipase et obésité
}

Les nouvelles
de ce numéro
ont été préparées par
Pascale Briand
Jean-Claude Dreyfus
Jean-Pierre Grünfeld
Axel Kahn
Jean-François Lacronique
Marc Peschanski
Jean Rosenbaum*

- Inserm V.99, hôpital Henri-Mondor, 51 avenue du Maréchal-de-Lattre-de-Tassigny, 94010 Créteil, France.

1. Kern PA, Ong JM, Saffari B, Carty J. The effects of weight loss on the activity and expression of adipose-tissue lipoprotein lipase in very obese humans. $N$ Engl J Med $1990 ; 322: 1053-9$.

2. Schwartz RS, Brunzell JD. Increase of adipose tissue lipoprotein lipase activity with weight loss. J Clin Invest 1981; 67: 1425-30.
Les mécanismes des troubles de la régulation lipidique qui mènent à l'obésité ne sont que partiellement connus. On tend à y faire jouer un rôle central à une enzyme, la lipoprotéine lipase (LPL). Dans les capillaires du tissu adipeux, la LPL hydrolyse les triglycérides liés aux protéines en acides gras libres. Ceux-ci sont repris par le tissu, réestérifiés, et mis en réserve dans les adipocytes. On a soulevé l'hypothèse que la LPL pourrait être à l'origine d'un signal de faim, notamment chez des rongeurs obèses, et on l'a désignée sous le terme de "portier de l'adipocyte". A plusieurs reprises, on a étudié l'activité de la LPL au cours des obésités humaines ; mais si l'on trouve bien une élévation chez les grands obèses, l'effet du régime amaigrissant a donné des résultats contradictoires. Kern et al. (Los Angeles, CA, USA) ont repris le problème [1] en s'efforçant de se placer dans les conditions les plus favorables : choix de sujets à forte obésité, six hommes et trois femmes pesant de 107 à $175 \mathrm{~kg}$ (moyenne $136 \mathrm{~kg}$ ) ; dosage de la LPL (activité et protéine immunoréactive) avant et après cure d'amaigrissement (perte de 16 à $66 \mathrm{~kg}$ ), sur des sujets à régime et poids stabilisés au moins pendant deux mois, avant comme après le prélèvement de tissu adipeux.

Le dosage de l'activité LPL a montré une augmentation chez tous les individus après la cure - en moyenne, un doublement - ainsi que de la protéine immunoréactive totale ; pour rechercher d'éventuels changements moléculaires post-traductionnels, de glycosylation par exemple, une électrophorèse suivie de révélation immunologique a été faite ; elle a montré une protéine de taille apparente $55 \mathrm{kDa}$, donc inchangée. L'analyse de l'ARN messager par Northern blot a révélé une augmentation d'environ deux fois sans changement de taille. C'est donc sans doute une élévation du messager qui est à l'origine de celle de la protéine, par hyperproduction transcriptionnelle ou stabilisation de l'ARNm. Il existe enfin une corrélation entre le degré de l'adiposité initiale et l'élévation de la LPL.

Avant de tirer de ces recherches une conclusion, il faut remarquer que d'autres travaux fournissent des résultats différents. Il est difficile de dégager les causes de ces variations. L'une d'elles est le régime au moment du deuxième examen; le facteur le plus important est probablement le degré d'obésité des sujets ; dans certains travaux, le poids initial était inférieur à $100 \mathrm{~kg}$; dans le seul article faisant état de poids comparables à ceux de Kern et al. [2], la LPL augmentait aussi après régime. Le degré de perte de poids importe également : si celle-ci est trop faible, la LPL ne réagit pas. Reste à établir les mécanismes de cette élévation de la LPL; ils sont probablement hormonaux; chez les obèses non traités, elle pourrait relever d'une hyperinsulinémie, mais avec la perte de poids l'insulinémie s'abaisse, et l'insuline ne doit pas être le seul régulateur de l'activité ou de la synthèse de la LPL.

En définitive, l'élévation de la LPL favorise la synthèse et l'accumulation des lipides dans les adipocytes. On conçoit qu'elle joue un rôle dans les difficultés que l'on éprouve, lors des traitements amaigrissants, à descendre audessous d'un certain poids et à en éviter la reprise. 